\title{
Off-Line Arabic Handwritten Characters Recognition Based on a Hidden Markov Models
}

\author{
M. Amrouch, M. Elyassa, A. Rachidi, and D. Mammass \\ IRF-SIC Laboratory \\ Faculty of Sciences, Agadir, Morocco \\ amrouch_mustapha@yahoo.fr, melyass@gmail.com, \\ rachidi@caramail.com, driss_mammass@yahoo.fr
}

\begin{abstract}
We present a system based on Hidden Markov Models (HMMs) for offline isolated Arabic handwritten characters recognition. The Hough accumulator of the character image is partitioned into equal horizontal bands that will be used to extract directional information. This information is translated into sequences of observations that are used to train the model for each character during the learning step.
\end{abstract}

Keywords: Isolated Arabic hand-written characters; Hough Transformation; Hidden Markov Models.

\begin{abstract}
Résumé
Nous présentons dans ce travail un système à base de Modèles de Markov cachée (HMMs) pour le problème de la reconnaissance hors-ligne des lettres arabes isolées manuscrites. L'accumulateur de Hough de l'image du caractère est partitionnée en bandes horizontales égales que nous utiliserons pour extraire une information directionnelle. L'information obtenue est traduite en séquences d'observations qui sont utilisées pour entraîner le modèle de chaque caractère lors de la phase d'apprentissage.
\end{abstract}

\section{Mots clés}

Caractères arabes isolés manuscrits, Transformation de Hough, Modèles de Markov Cachés.

\section{Introduction}

The morphology of the Arabic writing presents some characteristics which are the source of their treatments complexity. The Arabic writing is semi cursive script in its printed and handwritten forms. The characters of an Arabic word (or pseudo-word) are horizontally or vertically ligatured which darken the process of segmentation in characters. The forms of letters change according to their positions in the word. Besides, more than half of the Arabic characters include diacritic points in their shape [4] [5].

The Hidden Markov Models (HMMs) are among the most used methods in recognition ([1] [2] [3] [11] [12]). We present in this paper a Hidden Markov Models for off-line isolated Arabic handwritten characters recognition (see figure 1) which is distinguishes by the exploitation of the rates of presence of the dominant orientations of the characters Hough transform. 


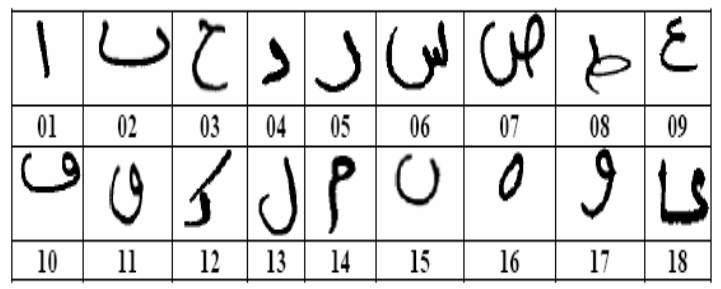

Fig. 1. Arab characters

In the section 2, we present the architecture of our system, the data base of Arabic handwritten characters and the pre-processing. The section 3 is reserved to the characteristics extraction and to the choice of the models, obtained from Markovian observations, which will represent the characters. The section 4 is dedicated to the learning and classification steps and the section 5 to the numerical results.

\subsection{Hidden Markov Models ( HMMs)}

A Hidden Markov Model is a double stochastic process with an underlying process which is not observable. This last one is only observed through another set of stochastic processes which produces the sequence of the symbols. An HMM is characterized by the state transition probabilities matrix $A$, the symbols probabilities of observation $B$ and the vector of probability of the initial state $\Pi$.

$N$ : The number of states;

$T$ : The number of observations or possible symbols;

$q_{t}:$ The state of the system at the time $\mathrm{t}$;

$M$ : Size of the observed sequence;

$A=\left\{a_{i j}=p\left(s_{j} / s_{i}\right)\right\} ; \sum_{\mathrm{j}=1}^{\mathrm{N}} \mathrm{a}_{\mathrm{ij}}=1$ is the matrix of the probability of transitions. $\Pi=\left\{\pi_{i}=p\left(s_{i}\right)\right\} ; \sum_{i=1}^{N} \pi_{i}=1$ is the vector of the initial probabilities.

$B=\left\{b_{i}\left(o_{k}\right)=p\left(o_{k} / s_{i}\right)\right\} ; \sum_{\mathrm{k}=1}^{\mathrm{T}} b_{\mathrm{i}}\left(o_{k}\right)=1$ are the probabilities of the observation symbols.

A Hidden Markov Model is characterized by a matrix of transition $A$, a matrix of observation $B$ and a vector of initialization $\Pi$.

We note: $\lambda=\{\Pi, A, B\}$

The use of the HMMs in the recognition is conditioned by the resolution of three problems: 
- Recognition: $\quad$ Let an $\operatorname{HMM} \lambda=\{\Pi, A, B\}$ and an observed sequence $O=\left\{o_{1}, o_{2}, \ldots o_{n}\right\}$, what is the value of the probability $p(O / \lambda)$ so that the model $\lambda$ generates $O$ ?

- Analysis an decoding : For an HMM and an observed sequence $O$, what is the sequence of the states which has the maximal probability to generated $O$ ?

- Learning: From a chain of observations $O=\left\{o_{1}, o_{2}, \ldots o_{n}\right\}$, how to adjust the parameters of the HMM $\lambda=\{\Pi, A, B\}$ to maximize $p(O / \lambda)$ ?

The solutions of these problems are well retailed in the literature [6] [7] [8] [9].

\section{System of Recognition}

\subsection{Architecture}

We begin with a pre processing step in order to remove noises due to the acquisition process of the characters (see figure 2). Directional primitives are extracted from the processed images by using the Hough transformation and then sequences of observations are generated. In the training step, we involve the HMM of each

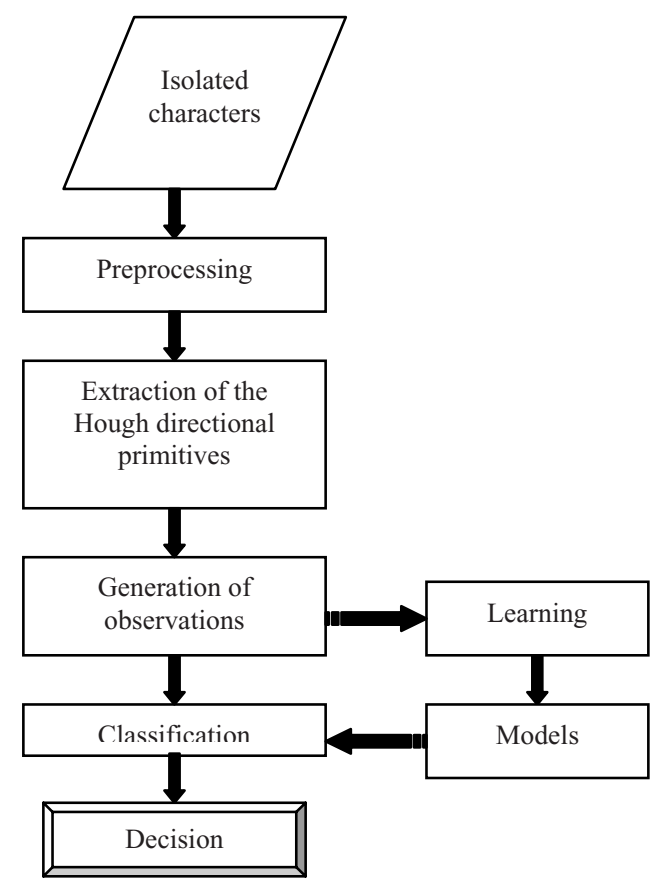

Fig. 2. System of recognition 
character, with a Baum-Welch process, by the various sequences of observations. The classification is done by the search for the characters discriminating models and we decide for the class of the character.

\subsection{Data Base}

The system is tested on a data base of 6188 isolated Arabic handwritten characters (26 persons giving, each one 14 times, 17 Arabic characters). The images of characters are in Gray level of dimensions 96 X 96 pixels. Some operators are used to reduce the noise and there is no operation of segmentation because we have only isolated forms.

\section{Extraction of Primitives}

The direct identification of the character from its image (matrix of pixels) seems very difficult because of the Arabic characters morphology and the variability in the used writing style. So, for each images, the Hough Transform [10] give a set of discriminating characteristics, from the character representation in pixels, that allows recognizing it easily. The algorithm of extraction is:

$\rightarrow$ Initialization of the table of the Hough Transform accumulator

$\rightarrow$ For each black pixel of the image $\left(x_{i}, y_{i}\right): 0 \leq x_{i} \leq n$ et $0 \leq y_{i} \leq m$ with $\mathrm{n}$ width image and $\mathrm{m}$ its height.

$\rightarrow 0 \leq \theta \prec 180^{\circ}$

$\rightarrow \rho_{k}=x_{i} \cos \theta_{k}+y_{i} \sin \theta_{k}$

$\rightarrow$ To increment the cell of the table of accumulator corresponding to the $\operatorname{couple}\left(\rho_{k}, \theta_{k}\right)$

The result of the algorithm on some characters is shown in figure 3.

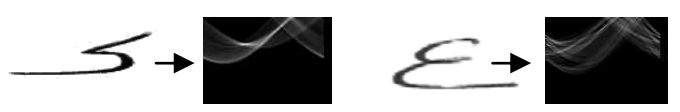

Fig. 3. Hough Transform on the characters KAF and AIN

In the experimentation, the images of characters are of fixed size: $96 \times 96$ pixels and the displacement $\Delta \theta$ is equal to 30 , consequently the Hough accumulator will contain information on the six orientations $\left(0^{\circ}, 30^{\circ}, 60^{\circ}, 90^{\circ}, 120^{\circ}\right.$ et $\left.150^{\circ}\right)$.

The images are cut out in 16 horizontal and 16 vertical bands and the intersections of these bands form 256 areas of dimension 6x6 pixels each one. For each area, we calculate the rates of the six orientations presence and we determine the dominant direction, and finally we memorize this information which will be used for the generation of observations. 


\subsection{Generation of the Directional Observations Sequences}

For the generation of the sequence of directional observations task, we select the minimum of the primitives representing the dominant directions. In each area, consider the rate of the dominant direction. Thereafter, we represent, by a symbol in the sequence of observations, a set of adjacent areas considered of the right-hand side towards the left and of the same rates. The symbols which can be emitted are 6 corresponding to the 6 basically orientations (see figure 4).

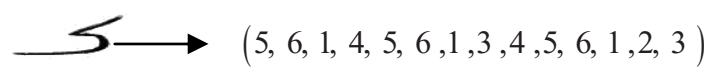

Fig. 4. Sequence of the character KAF

\subsection{Choice of Models}

The images are translated into sequences of observations which give the Hidden Markov Models representing every character. In the literature, various models and architectures were proposed for the use of the HMM in the handwritten writing recognition ([11][12]). In our work, we opted for a right-left topology where the number of states is strictly lower than the number of horizontal bands of the character. Indeed, the bands of the extremities supply no observation.

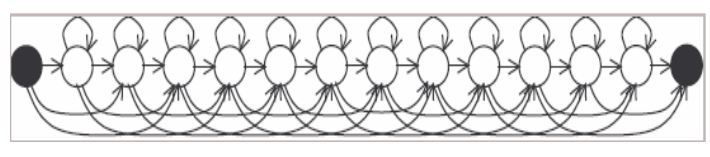

Fig. 5. Model of character with $N$ states [3]

\section{Learning and Classification}

\subsection{Learning}

We train the hidden Markov models of various characters by the classical procedure of Baum-Welch to adjust their parameters. Every character possesses its own model. Consequently, the algorithms look for, in the space of the HMM modelling of each character, the model which has the maximum probability to generate the sequence of observations "generation of the sequence of observation ". The best HMM is selected to form a base of learning (see figure 5).

\subsection{Classification}

In the learning step, we obtain so many HMMs as there were of characters. The classification is made at first by the search of the discriminating model among every 


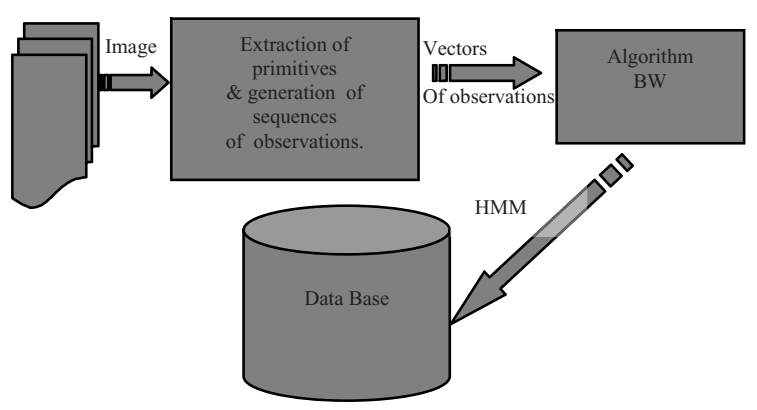

Fig. 6. Scheme of the learning data

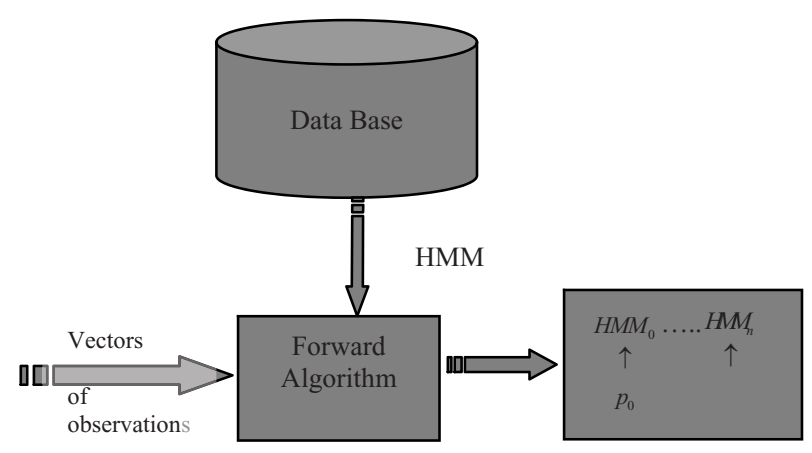

Fig. 7. Scheme of the classification process

selected HMMs of each character. We calculate, by the Forward algorithm, the probability such that the models can generate the sequence of observations of the character to be recognized. Afterward, we have a set of models where every model is associated with a score. The elected model is the one possessing the biggest score (see figure 6).

\section{Numerical Results}

We used 42 Arabic handwritten isolated characters (14 KAF, 14 HA, 14 AIN) extracted from the base of characters. $50 \%$ served for the phase of learning, and $50 \%$ for the tests. Encouraging results appear with a rate of $85.71 \%$ of good recognition. The best score was $100 \%$ for the character (Ha) (see figure 8 ).

The errors of recognition are big for the letter AIN, what is explained by the incapacity of the used characteristics to describe every character (very big variability in the styles of the writing of this character). A good estimation of these data can decrease the rate of error of our system. 


\begin{tabular}{|c|c|c|}
\hline $\begin{array}{c}\text { Arabic } \\
\text { character }\end{array}$ & $\begin{array}{c}\text { Translation } \\
\text { in French }\end{array}$ & $\begin{array}{c}\text { Rate of } \\
\text { recognition }\end{array}$ \\
\hline & $\mathrm{Ha}$ & $100 \%$ \\
\hline 5 & $\mathrm{KAF}$ & $85,71 \%$ \\
\hline$\varepsilon$ & AIN & $71,42 \%$ \\
\hline
\end{tabular}

Fig. 8. Example of rate recognition

\subsection{Conclusion}

In this paper, we approached the problem of the off-line isolated Arabic handwritten characters recognition based on Hidden Markov Models (HMMs) and directional information from the Hough transformation of every character.

The results are promising and show that the Hidden Markov Models are well adapted to the variation in the length of the handwritten writing. However, its capacity of discrimination is not very high because every HMM uses the data of learning of a single character. One of the weaknesses of the HMMs, results from the level of the probability estimation of the emission of observations. To ovoid these problems, we think to use an hybrid method combining the HMMs and the neural networks.

\section{References}

[1] Belaïd, A., Saon, G.: Utilisation des processus markoviens en reconnaissance de l'écriture. Traitement de signal 14(2), 161-178 (1997)

[2] El Yacoubi, A.: Modélisation markovienne de l'écriture manuscrite, application à la reconnaissance des adresses postales. Thèse de doctorat, Université de Rennes 1 (1996)

[3] poisson, E.: Architecture et Apprentissage d'un système hybride de Neuro-Markovien pour la reconnaissance de l'écriture Manuscrite en-ligne, Thèse de doctorat, unversité de Nantes (2005)

[4] Ben Amara, N., Belaïd, A., Ellouze, N.: Modélisation pseudo bidimensionnelle pour la reconnaissance de chaînes de caractères arabes imprimés. In: Proc. 1er Colloque international francophone sur l'écrit et le document (CIFED 1998), Québec, Canada, pp. 131-140 (1998)

[5] Miled, H. : Stratégies de reconnaissance de l'écriture semi cursive : application aux mots manuscrits arabes. Thèse de doctorat, Université de Rouen (1998)

[6] Cornuéjols, A., Miclet Eyrolles, L.: Apprentissage Artificiel : Méthodes et Algorithmes' IRISA, ENSSAT (2002)

[7] Dupré, X.: Contributions à la reconnaissance de l'écriture cursive à l'aide de modèles de Markov cachés, Thèse de doctorat, Université René Descartes - Paris V (2004)

[8] Korilis, Y., St-Jean, C., DeBarr, D., Carpenter, B., Chu-Carroll, J.: Modèles de Markov Cachés

[9] Agen, F., Michot, J.: Chaînes de Markov cachées Algorithme de Baum-Welch, Ecole polytechnique université de Tours (2004-2007) 
[10] Maitre, H.: Un panorama de la transformation de Hough. In: Traitement de signal, vol. 2 (1985)

[11] Mahjoub, M.A.: Application des modèles de Markov Cachés stationnaires et non stationnaires et non stationnaires à la reconnaissance en ligne de l'écriture arabe, Thèse de doctorat ENIT (1999)

[12] Touj, S., Touj, S., Ben Amara, N., Amiri, H.: Reconnaissance Hors Ligne de Caractères Arabes Isolés Manuscrits, Institut Supérieure des Etudes Technologiques de Gabès, Ecole Nationale d'Ingénieurs de Monastir, Ecole Nationale d'Ingénieurs de Tunis, CIFED 2002 (2002) 